\title{
Information-Theoretic Approach to Steganographic Systems
}

\author{
Boris Ryabko \\ Institute of Computational Technologies \\ of Siberian Branch of Russian Academy of Science, \\ Siberian State University of Telecommunications and Informatics, \\ Novosibirsk, Russia; boris@ ryabko.net
}

\author{
Daniil Ryabko \\ IDSIA, Switzerland \\ daniil@ryabko.net
}

\begin{abstract}
We propose a simple universal (that is, distributionfree) steganographic system in which covertexts with and without hidden texts are statistically indistinguishable. The stegosystem can be applied to any source generating i.i.d. covertexts with unknown distribution, and the hidden text is transmitted exactly, with zero probability of error. Sequences of covertexts with and without hidden information obey the same distribution (the stegosystem is perfectly secure). The proposed steganographic system has two important properties. First, the rate of transmission of hidden information approaches the Shannon entropy of the covertext source as the size of blocks used for hidden text encoding tends to infinity. Second, if the size of the alphabet of the covertext source and its minentropy tend to infinity then the number of bits of hidden text per letter of covertext tends to $\log (n !) / n$ where $n$ is the (fixed) size of blocks used for hidden text encoding. Besides, the resource complexity of the proposed algorithms grows only polynomially.
\end{abstract}

\section{INTRODUCTION}

The goal of steganography is as follows. Alice and Bob can exchange messages of a certain kind (called covertexts) over a public channel which is open to Eve. The covertexts can be, for example, a sequence of photographic images, videos, text emails and so on. Alice wants to pass some secret information to Bob so that Eve cannot notice that any hidden information was passed. Thus, Alice should use the covertexts to hide the secret text. Alice and Bob may share a secret key. A classical illustration from [12] states the problem in terms of communication in a prison: Alice and Bob are prisoners who want to concoct an escape plan passing each other messages which can be read by a ward.

We assume that Eve does not attempt to disrupt communication between Alice and Bob, but only tries to determine whether secret information is being passed.

Perhaps the first formal approach to steganography was taken by Cachin [1], [2] who proposed a steganographic protocol in which, relying on the fact that the probability distribution of covertexts is known, covertexts with and without hidden information are statistically close. In the same work a universal (distribution-free) steganographic system was proposed, in which this property holds only asymptotically with the size of the messages going to infinity, and which has exponential complexity of coding and decoding.

Distribution-free stegosystems are of particular practical importance, since in reality covertexts can be a sequence of graphical images, ICQ or email messages, that is, sources for which the distribution is not only unknown but perhaps cannot be reasonably approximated.

In the context of a known covertext distribution, theoretical capacity of perfectly secure stegosystems (that is, stegosystems in which covertext with and without hidden information are statistically indistinguishable) was analyzed in [8], [5].

We consider an information-theoretic approach, where "statistically indistinguishable" means that the covertexts with and without hidden information have the same probability distribution. In other words, Eve does not get any information for distinguishing different kinds of the covertexts. It is worth noting that, since Shannon's celebrated paper "Communication theory of secrecy systems" [11], the information-theoretic approach was efficiently applied to many problems of secrecy systems, see e.g. [6] and references therein.

We use the following model for steganography, mainly following [2]. It is assumed that Alice has an access to an oracle which generates independent and identically distributed covertexts according to some fixed but unknown distribution $\mu$. Covertexts belong to some (possibly infinite) alphabet $A$. Alice wants to use this source for transmitting hidden messages. A hidden message is a sequence of letters from $B=\{0,1\}$ generated independently with equal probabilities of 0 and 1 . We denote the source of hidden messages by $\omega$. This is a commonly used model for the source of secret messages since it is assumed that secret messages are encrypted by Alice using a key shared only with Bob. If Alice uses the Vernam cipher then the encrypted messages are indeed generated according to the Bernoulli $1 / 2$ distribution, whereas if Alice uses modern block or stream ciphers then the encrypted sequence "looks like" a sequence of random Bernoulli $1 / 2$ trials. (Here to "look like" means to be indistinguishable in polynomial time, or that the likeness is confirmed experimentally by statistical data, known for all widely used cyphers; see e.g. [7], [9].) The third party, Eve, is reading all messages passed from Alice to Bob and is trying to determine whether secret messages are being passed in the covertexts or not. Observe that if covertexts with and without hidden information have the same probability distribution $(\mu)$ then it is impossible to distinguish them.

We propose a simple universal perfectly secure stegosystem; that is, covertexts with and without hidden information have 
the same distribution (and hence are statistically indistinguishable) for any size of the message, for any source of i.i.d. covertexts. The hidden text is transmitted correctly with probability 1 . Moreover, the proposed system has two important properties. First, the rate of transmission of hidden information approaches the Shannon entropy of the covertext source as the size $n$ of blocks used for hidden text encoding tends to infinity. Second, if the size of the alphabet of the covertext source and its minentropy tend to infinity then the number of bits of hidden text per letter of covertext tends to $\log (n !) / n$ where $n$ is the (fixed) size of blocks used for hidden text encoding. We note that it is also possible to use the proposed stegosystems for open-key steganography, since the steganographic protocol does not require any secret key.

The paper is organized as follows. In Section II a simple stegosystem which does not use randomization is proposed; for this system the number of bits of hidden text per letter of covertext tends to $1 / 2$ if the size of the alphabet of the covertext source and its minentropy tend to infinity. This system also illustrates the main ideas used in Section III, where the general (randomized) stegosystem is proposed which has the mentioned asymptotic properties of the rates of hidden text transmission. In Section IV we discuss possible extensions of the proposed steganographic systems and outline some potentially interesting open problems.

\section{A SIMPLE NON-RANDOMIZED UNIVERSAL STEGOSYSTEM}

In this section we present a very simple stegosystem which demonstrates the main ideas used in the general stegosystem which we develop in the next section. The stegosystem described in this section does not use randomization.

The notation is as follows. The source $\mu$ draws i.i.d. (covertext) letters from an alphabet $A$. The source $\omega$ draws i.i.d. (hidden, or secret) equiprobable letters from the alphabet $B=\{0,1\}$. Elements of $A(B)$ are usually denoted by $x(y)$.

First consider an example. Consider a situation in which not only the secret letters are drawn (using $\omega$ ) from a binary alphabet, but also the source of covertexts $\mu$ generates symbols from the alphabet $A=\{a, b\}$ (not necessarily with equal probabilities). Suppose that Alice has to transmit the sequence $y^{*}=y_{1} y_{2} \ldots$ generated according to $\omega$ and let there be given a covertext sequence $x^{*}=x_{1} x_{2} \ldots$ generated by $\mu$. For example, let

$$
y^{*}=01100 \ldots, \quad x^{*}=a a b a b a a a a b b a a a a a b b \ldots .
$$

The sequences $x^{*}$ and $y^{*}$ are encoded in a new sequence $X$ (to be transmitted to Bob) such that $y^{*}$ is uniquely determined by $X$ and the distribution of $X$ is the same as the distribution of $x^{*}$ (that is, $\mu$; in other words, $X$ and $x^{*}$ are statistically indistinguishable).

The encoding is carried out in two steps. First let us group all symbols of $x^{*}$ into pairs, and denote

$$
a a=u, b b=u, a b=v_{0}, b a=v_{1} .
$$

In our example, the sequence (1) is represented as

$$
x^{*}=a a b a b a a a a b b a a a a a b b \cdots=u v_{1} v_{1} u v_{0} v_{1} u u u \ldots
$$

Then $X$ is obtained from $x^{*}$ as follows: all pairs corresponding to $u$ are left unchanged, while all pairs corresponding to $v_{k}$ are transformed to pairs corresponding to $v_{y_{1}} v_{y_{2}} v_{y_{3}} \ldots$; in our example

$$
X=a a a b b a a a b a a b a a a a b b \ldots
$$

Decoding is obvious: Bob groups the symbols of $X$ into pairs, ignores all occurrences of $a a$ and $b b$ and changes $a b$ to 0 and $b a$ to 1 .

The properties of the described stegosystem, which we call $S t_{2}$, are summarized in the following (nearly obvious) statement.

Claim 1: Suppose that a source $\mu$ generates i.i.d. random variables taking values in $A=\{a, b\}$ and let this source be used for encoding secret messages consisting of a sequence of i.i.d. equiprobable binary symbols using the method $S t_{2}$. Then the sequence of symbols output by the stegosystem obeys the same distribution $\mu$ as the input sequence.

We will not give the (obvious) proof of this claim since it is a simple corollary of Theorem 1 below.

It is interesting to note that a similar construction was used by von Neumann in his method for obtaining a sequence of equiprobable binary symbols (see [13], [3]) from a sequence of independent flips of a biased coin. His method, as well as the stegosystem just described, was based on the fact that the probabilities of $a b$ and $b a$ are equal.

Next we consider the generalisation of the described stegosystem to the case of an arbitrary alphabet $A$ (such that $|A|>1$ ). To do this we fix some total ordering on the set $A$. As before, Alice has to transmit a sequence $y^{*}=y_{1} y_{2} \ldots$ generated by the source $\omega$ of i.i.d. equiprobable binary letters and let there be given a sequence $x^{*}=x_{1} x_{2} \ldots$ of covertext letters generated i.i.d. according to a distribution $\mu$ on $A$. Again we transform the sequences $y^{*}$ and $x^{*}$ into a new sequence $X$ which obeys the same distribution as $x^{*}$. As before we break $x^{*}$ into blocks of length 2 . If a block $x_{2 i-1} x_{2 i}$ has the form $a a$ for some $a \in A$ then it is left unchanged. Otherwise let the block $x_{2 i-1} x_{2 i}$ be $a b$ for $a, b \in A$ and suppose $a<b$; if the current symbol $y_{k}$ is 0 then the block $a b$ is included in $X$, and if $y_{k}=1$ then $b a$ is included in $X$. If $a>b$ then encode in the opposite way. To decode, the sequence is broken into pairs of symbols, all pairs of the form $a a$ are ignored and a pair of the form $a b$ is decoded as 0 if $a<b$ and as 1 otherwise. Denote this stegosystem by $S t_{2}(A)$.

Theorem 1: Suppose that a source $\mu$ generates i.i.d. random variables taking values in some alphabet $A$. Let this source be used for encoding secret messages consisting in a sequence of i.i.d. equiprobable binary symbols, using the method $S t_{2}(A)$. Then the sequence of symbols output by the stegosystem obeys the same distribution $\mu$ as the input sequence and the number of letters of hidden text transmitted per letter of covertext is $\frac{1}{2}\left(1-\sum_{a \in A} \mu(a)^{2}\right)$. 
Proof: Fix some $\alpha, \beta \in A$ and $k \in \mathbb{N}$. We will show that

$$
p\left(X_{2 k-1} X_{2 k}=\alpha \beta\right)=\mu(\alpha \beta),
$$

where $p$ is the probability distribution of the output sequence. Suppose $\alpha<\beta$. Decomposing the probability on the left we get

$$
\begin{aligned}
p\left(X_{2 k-1} X_{2 k}=\alpha \beta\right)= & \omega\left(y_{k}=0\right)(\mu(\alpha \beta)+\mu(\beta \alpha)) \\
& =\frac{1}{2}(\mu(\alpha \beta)+\mu(\alpha \beta))=\mu(\alpha \beta) .
\end{aligned}
$$

The case $\beta<\alpha$ is analogous, and the case $\beta=\alpha$ is trivial. The second statement is obtained by calculating the probability that letters in the block coincide.

Note that in practice when the covertexts are, for example, graphical files, each covertext is practically unique (the alphabet $A$ is potentially infinite) so that the number of covertext letters (files) per one hidden bit is approximately 2 .

\section{General CONSTRUCTION OF A UNIVERSAL STEGOSYSTEM}

In this section we consider the general construction of universal stegosystem which has the desired asymptotic properties. As before, Alice needs to transmit a sequence $y^{*}=$ $y_{1} y_{2} \ldots$ of secret binary messages drawn from an i.i.d. source $\omega$ with equal probabilities of 0 and 1 , and let there be given a sequence of covertexts $x^{*}=x_{1} x_{2} \ldots$ drawn i.i.d. from a source $\mu$ with alphabet $A$. First we break the sequence $x^{*}$ into blocks of $n$ symbols each, where $n>1$ is a parameter. Each block will be used to transmit several symbols from $y^{*}$ (for example, in the previously constructed stegosystem $S t_{2}(A)$ each block of length 2 was used to transmit 1 or 0 symbols). However, in the general case a problem arises which was not present in the construction of $S t_{2}(A)$. Namely, we have to align the lengths of the blocks of symbols from $x^{*}$ and from $y^{*}$, and for this we will need randomization. The problem is that the probabilities of blocks from $y^{*}$ are divisible by powers of 2 , which is not necessarily the case with blocks from $x^{*}$.

We now present a formal description. Let $u$ denote the first $n$ symbols of $x^{*}: u=x_{1} \ldots x_{n}$, and let $\nu_{u}(a)$ be the number of occurrences of the symbol $a$ in $u$. Define the set $S_{u}$ as consisting of all words of length $n$ in which the frequency of each letter $a \in A$ is the same as in $u$ (the set of all words that have the same type as $u$ ):

$$
S_{u}=\left\{v \in A^{n}: \forall a \in A \nu_{v}(a)=\nu_{u}(a)\right\} .
$$

Observe that the $\mu$-probabilities of all members of $S_{u}$ are equal. Let there be given some ordering on the set $S_{u}$ (for example, lexicographical) which is known to both Alice and Bob (and to anyone else) and let $S_{u}=\left\{s_{0}, s_{1}, \ldots, s_{\left|S_{u}\right|-1}\right\}$ with this ordering.

Denote $m=\left\lfloor\log _{2}\left|S_{u}\right|\right\rfloor$, where $\lfloor y\rfloor$ stands for the largest integer not greater than $y$. Consider the binary expansion $\left(\alpha_{m}, \alpha_{m-1}, \ldots, \alpha_{0}\right)$ of $\left|S_{u}\right|$, where $\alpha_{m}=1, \alpha_{j} \in\{0,1\}$, $m>j \geq 0$. In other words,

$$
\left|S_{u}\right|=2^{m}+\alpha_{m-1} 2^{m-1}+\alpha_{m-2} 2^{m-2}+\ldots+\alpha_{0} .
$$

Define a random variable $\Delta$ as taking each value $i \in$ $\{0,1, \ldots, m\}$ with probability $\alpha_{i} 2^{i} /\left|S_{u}\right|$ :

$$
p(\Delta=i)=\alpha_{i} 2^{i} /\left|S_{u}\right| .
$$

Alice, having read $u$, generates a value of the random variable $\Delta$, say $d$, and then reads $d$ symbols from $y^{*}$. Consider the word $r^{*}$ represented by these symbols as an integer which we denote by $r$. Then we encode the word $r^{*}$ (that is, $d$ bits of $y^{*}$ ) by the word $s_{\tau}$ from the set $S_{u}$, where

$$
\tau=\sum_{l=d+1}^{m} \alpha_{l} 2^{l}+r
$$

(In other words, the word $s_{\tau}$ is being output by the coder.)

Then Alice reads the next $n$-bit word, and so on. Denote the constructed stegosystem by $S t_{n}(A)$.

To decode the received sequence Bob breaks it into blocks of length $n$ and repeats all the steps in the reversed order: by the current word $u$ he obtains $S_{u}$ and $\tau$, then $d$ (clearly $d$ is uniquely defined by $\tau), r$ and $r^{*}$; that is, he finds $\left|r^{*}\right|$ next symbols of the secret sequence $y^{*}$.

Consider an example which illustrates all the steps of the calculation. Let $A=\{a, b, c\}, n=3, u=b a c$. Then $S_{u}=$ $\{a b c, a c b, b a c, b c a, c a b, c b a\},\left|S_{u}\right|=6, m=2, \alpha_{2}=1, \alpha_{1}=$ $1, \alpha_{0}=0$. Let the sequence of secret messages be $0110 \ldots$, that is, $y^{*}=0110 \ldots$. Suppose the value of $\Delta$ generated by Alice is 1 . Then she reads one symbol of $y^{*}$ (in this case 0 ) and calculates $r=0, r^{*}=0, \tau=2^{2}+0=4$ and finds the codeblock $s_{4}=c a b$. To decode the message, Bob from the block $c a b$ calculates $\tau=4, r=0, r^{*}=0$ and finds the next symbol of the secret sequence -0 .

Theorem 2: Let a source $\mu$ be given, which generates i.i.d. random variables taking values in some alphabet $A$. Let this source be used for encoding secret messages consisting of a sequence of i.i.d. equiprobable binary symbols using the described method $S t_{n}(A)$ with $n>1$. Then

(i) the sequence of symbols output by the stegosystem obeys the same distribution $\mu$ as the input sequence,

(ii) the average number of secret symbols per covertext $\left(L_{n}\right)$ satisfies the following inequality

$$
L_{n} \geq \frac{1}{n}\left(\sum_{u \in A^{n}} \mu(u) \log \frac{n !}{\prod_{a \in A} \nu_{u}(a) !}-2\right),
$$

where $\mu(u)$ is the $\mu$-probability of the word $u$ and $\nu_{u}(a)$ is the number of occurrences of the letter $a$ in the word $u$.

Proof: To prove the first statement it is sufficient to show that for any covertext word $u$ of length $n$ its probability of occurrence in the output sequence is $1 /\left|S_{u}\right|$. This follows from (2) and the fact that letters in $y^{*}$ are independent and equiprobable.

The second statement can be obtained by direct calculation of the average number of symbols from $y^{*}$ encoded by one block. Indeed, from (2) we find that for each covertext word $u$ the expected number of transmitted symbols is 
$\frac{1}{\left|S_{u}\right|} \sum_{l=1}^{m} l \alpha_{l} 2^{l} \geq\left|S_{u}\right|-2$, where $m=\left\lfloor\log _{2}\left|S_{u}\right|\right\rfloor$, and for each word $u$ we have

$$
\left|S_{u}\right|=\frac{n !}{\prod_{a \in A} \nu_{u}(a) !} .
$$

Let us now consider the asymptotic behaviour of $L_{n}$ when $n \rightarrow \infty$.

Corollary 1: If the alphabet $A$ is finite then the average number of hidden symbols per letter $L_{n}$ goes to the Shannon entropy $h(\mu)$ of the source $\mu$ as $n$ goes to infinity; here by definition $h(\mu)=-\sum_{a \in A} \mu(a) \log \mu(a)$.

Proof: This statement follows from a well-known fact of Information Theory which states that for each $\delta>0$ and $n \rightarrow \infty$ the following inequality holds with probability 1

$$
h(\mu)-\delta<\log \left|S_{u}\right| / n<h(\mu)+\delta
$$

see e.g. [4].

In many real stegosystems the alphabet $A$ is huge (it can consist, for example, of all possible digital photographs of given file format, or of all possible e-mail messages). In such a case it is interesting to consider the asymptotic behaviour of $L_{n}$ with fixed $n$ when the alphabet size $|A|$ goes to infinity. For this we need to define the so-called min-entropy of the source $\mu$ :

$$
H_{\infty}(\mu)=\min _{a \in A}\{-\log \mu(a)\}
$$

Corollary 2: Assume the conditions of Theorem 2 and fix the block length $n>1$. If $|A| \rightarrow \infty$ so that $H_{\infty}(\mu) \rightarrow \infty$ then $L_{n}$ tends to $(\log (n !)-O(1)) / n$.

Proof: This statement simply follows from the fact that the number of different permutations of $n$ elements is $n$ !.

When the alphabet size is supposed to be very large another parameter of a stegosystem becomes important, namely the number of draws from the oracle distribution. In this context we note that the number of symbols per transmitted covertext in the proposed stegosystem is also the number of symbols per generated covertext; that is, no extra draws from the oracle are necessary.

Next we briefly consider the resource complexity of the stegosystem $S t_{n}(A)$. To store all possible words from the set $S_{u}$ would require memory of order $2^{n} \log |A|$ bits, which is practically unacceptable for large $n$. However, if we use the algorithm for fast enumeration from [10], then we can find the index of a block $s_{\tau}$ given $\tau$ (encoding) and vice versa (decoding) using $O\left(\log ^{\text {const }} n\right)$ operations per symbol and $O\left(n \log ^{3} n\right)$ bits of memory.

\section{Discussion}

We have proposed two stegosystems (with and without randomization) for which the output sequence of covertexts with hidden information is statistically indistinguishable from a sequence of covertexts without hidden information. The proposed stegosystems rely heavily on the assumption that the oracle generates independent and identically distributed covertexts. This is perhaps a reasonable assumption if a covertext is a sequence of graphical images of a certain kind, but if, for example, we want to use just one image to transmit (a large portion of) a secret text then our covertexts are parts of the image, which are clearly not i.i.d. How to extend the ideas developed in this work to the case of non-i.i.d. covertexts is perhaps the main open question.

However, the main idea that was used in the proposed stegosystems is that for any block of covertexts it is possible to find several other blocks which have the same probability as the original one; then hidden information can be encoded in the number of a block in this group. This idea can be extended to the case of non-independent covertexts. Indeed, suppose that on the current step of transmission we known that some covertexts have equal probabilities to appear as the next generated covertext. That is, among the conditional (given the current history) probabilities of covertexts there are several groups of equal probabilities. Then, if the probability of the next generated covertext belongs to one of these groups, we can use this covertext (possibly replacing it with another one which has the same probability) for encoding several next bits of hiddentext in the same fashion as it is done in $S t_{n}(A)$. The same applies to blocks of covertexts. Indeed the only feature of independently and identically distributed covertexts that we use is that all permutations of a word of size $n$ have equal probabilities. So the next step is to identify equal conditional probability groups in sources of non-i.i.d. covertexts.

Acknowledgment: Boris Ryabko was supported by Russian Foundation for Basic Research (grant no. 06-07-89025). Daniil Ryabko was supported by the Swiss NSF grants 200020107616 and 200021-113364.

\section{REFERENCES}

[1] C. Cachin, "An information-theoretic model for steganography," In: Proc. 2nd Information Hiding Workshop, vol. 1525 of LNCS, pp. 306-318, Springer Verlag, 1998.

[2] C. Cachin, "An information-theoretic model for steganography," Information and Computation, vol. 192, pp. 41-56, 2004

[3] P. Elias, "The Efficient Construction of an Unbiased Random Sequence," The Annals of Mathematical Statistics vol. 43 (3), pp. 864-870, 1972.

[4] R.G. Gallager, "Information Theory and Reliable Communication," John Wiley \& Sons, New York, 1968.

[5] J. Harmsen and W. Pearlman, "Capacity of steganographic channels", Proceedings 7th ACM Workshop on Multimedia and Security, 2005.

[6] U. Maurer "Information-Theoretic Cryptography," In: Proc. Advances in Cryptology CRYPTO 1999, LNCS vol. 1666,, Springer-Verlag, pp. 47-64, 1999.

[7] A. Menzes, P. van Oorschot, S. Vanstone "Handbook of Applied Cryptography". CRC Press, 1996.

[8] P. Moulin and Y. Wang, "New results on steganographic capacity", —em Proceedings Conference on Information Science and Systems, Princeton, New Jersey, 2004.

[9] B. Ryabko, A. Fionov, "Basics of Contemporary Cryptography for IT Practitioners", World Scientific Publishing Co., 2005.

[10] B. Ryabko, "Fast enumeration of combinatorial objects", Discrete Mathematics and Applications, vol. 10, no. 2, 1998. (see also http://arxiv.org/abs/cs.CC/0601069)

[11] C.E. Shannon, "Communication theory of secrecy systems", Bell Sys. Tech. J., vol. 28, pp. 656-715, 1948.

[12] G.J. Simmons, "The Prisoner's Problem and the Subliminal Channel" In: Proceedings of CRYPTO'83, 1984.

[13] J. von Neumann "Various Techniques Used in Connection with Random Digits," Monte Carlo Method, Applied Mathematics Series, no. 12, U.S. National Bureau of Standarts, Washington D.C., pp. 36-38, 1951. 